\title{
Clinico-Pathological Profile and Management Outcome of Fournier's Gangrene in a Tertiary Care Hospital
}

\author{
*ME Ullah ${ }^{1}$, A Faruk ${ }^{2}$, RH Talukder ${ }^{3}$, Farukuzzaman $^{4}$, NA Alam ${ }^{5}$
}

\begin{abstract}
Background: Fournier's gangrene is a vascular disaster of infective origin occurring about male external genitalia. Diabetes Mellitus is an important predisposing factor. A good number of patients of this rare clinical entity are admitted frequently in our centre. Till now the disease has the potential for significant morbidity and also mortality.
\end{abstract}

Objective: The study was designed to be conducted among patients suffering from Fournier's gangrene to identify the possible source of sepsis, to denote pattern and extent of area involvement and microorganisms isolated and to observe the treatment course and outcome.

Methods: This prospective observational study was carried out in BIRDEM General Hospital, Dhaka among 60 patients of Fournier's gangrene admitted under surgery department during the period of October 2016 to January 2018 using purposive sampling method.

Result: The results of this study suggest that majority of the study population were in 51 to 60 years (Mean $52 \pm 4.9)$ age group. All the patients were diabetic with grossly elevated blood glucose level (Mean $\mathrm{RBS}=17.3 \mathrm{mmol} / \mathrm{dl}$ ). In addition to scrotum, perineum, penis, buttock and lower abdomen were also affected to variable extent $(73.3 \%)$. All patients required major debridement which had to be repeated in $68.3 \%$ cases. Steptococcus pyogens was the most common organism isolated $(43.3 \%)$ followed by Eschericia coli (35\%) and majority were of polymicrobial type (61.6\%). Mortality rate was $8.3 \%$.

Conclusion: The diagnosis and treatment of Fournier's gangrene is enigmatic and challenging to the physician. Urgent surgical debridement along with other appropriate supportive measures can reduce morbidity and mortality.

Key Word: Fournier's gangrene, Necrotizing fasciitis

\section{Introduction}

Fournier's gangrene is a rapidly progressive infection of the male external genital, perineal and perianal region with occasional cranial extension to the lower abdominal wall. It is a nasty infective disaster of vascular origin. It is characterized by a synergistic, necrotizing fasciitis (NF) leading to the thrombotic occlusion of small subcutaneous vessels and the development of gangrene. ${ }^{1}$ Often the initiating event is idiopathic.

*Dr. Mahmud Ekram Ullah, Dept. of Surgery, BIRDEM General Hospital, Shahbag, Dhaka-1000, Bangladesh Dr. Amreen Faruk, Dept. of Surgery, BIRDEM General Hospital, Shahbag, Dhaka-1000, Bangladesh

Dr. Rajibul Haque Talukder, Dept. of Surgery, BIRDEM General Hospital, Shahbag, Dhaka-1000, Bangladesh

Dr. Farukuzzaman, Dept. of Surgery, BIRDEM General Hospital, Shahbag, Dhaka-1000, Bangladesh

Dr. Noor A Alam, Dept. of Surgery, BIRDEM General Hospital, Shahbag, Dhaka-1000, Bangladesh

*Corresponding Author

Date of submission: 23.03.2018, Date of acceptance: 05.05.2018

AKMMC J 2018; 9(2) : 124-130 
proposed by Smith et $a l^{4}$ as an infective necrotizing fasciitis of the perineal, genital and perianal region. The major sources of sepsis are local skin, colon, anus, rectum and lower urinary tract.

The majority of patients with Fournier's gangrene are immunocompromised and thus the primary wound might have been minor or might have arisen from an otherwise uneventful surgery. ${ }^{5}$ Diabetes mellitus, advanced malignant disease, obesity, peripheral vascular disease, local trauma, urethral stricture and perianal disease have been cited as the main predisposing factors. ${ }^{6}$

At the initial stages of presentation, it has a paucity of clinical signs and is difficult to differentiate it from cellulitis. A high index of suspicion is needed to diagnose it. According to the microbiological characteristics, NF is classified into Type 1 (synergistic polymicrobial infections including anaerobes) and Type 2 (mono microbial infections), the former being more common. ${ }^{7}$ The most common mono microbial infection causing organisms include Beta-hemolytic Streptococcus, Staphylococcus aureus, and Clostridial species. Common poly microbial synergistic infection causing organisms includes S. aureus, Streptococcus pyogenes, Enterococci species, Escherichia coli, Pseudomonas species, and anaerobic organisms such as Bacteroides. $^{8}$

The precise pathogenesis of Fournier's gangrene is unclear. Inoculation of microbes can occur through minor trauma, insect bite, boil, perianal and perineal surgery etc. Under favorable environmental conditions such as immune compromised states, diabetes mellitus, liver failure and renal failure, organisms multiply to commence disease process. ${ }^{9}$ The process at first starts in deep tissue plane, so superficial skin signs may not be evident initially. This may lead to delay in diagnosis of this condition. Some patients present with toxic features due to sepsis without any/minimal underlying signs. Later, they may develop edema, erythema, tenderness, bullae, gangrenous skin patches and crepitus. ${ }^{10}$

NF in general is usually diagnosed by clinical features, but other investigations may help to confirm it. Plain X-ray may show subcutaneous gas. Computed tomography scan and magnetic resonance image may show asymmetrical fascial thickening, fat stranding, and gas tracking along fascial planes. ${ }^{11}$ Tissue biopsy reveals necrosis, polymorph nuclear infiltration and thrombosis of vessels. The management includes initial resuscitation, supportive care, adequate control of risk factors such as blood sugars; extensive debridement as early as possible which may have to be repeated, intravenous antibiotics and blood transfusion. ${ }^{9}$ The mortality rates of NF have remained alarmingly high with reported mortality rates ranging from $20 \%$ to $30 \% .{ }^{11}$ Studies have shown that delay in the diagnosis and consequently delayed operative debridement has caused increase in the mortality. ${ }^{10}$

Fournier's gangrene occurs worldwide as well as in our country. Although there is scarcity of statistical data regarding it's incidence in our country, we regularly get a good number of patients in our hospital. The purpose of this study is to look at Fournier's gangrene in terms of its clinical consequences and microbiological characteristics.

\section{Materials and Method}

During the study period, a total of 60 patients with Fournier's gangrene were admitted to surgery department of BIRDEM general Hospital. Thorough clinical assessment and relevant investigations were done. Diagnosis in all the cases was made on clinical basis. Urgent resuscitation in appropriate cases with fluid with or without blood transfusion and IV antibiotic therapy was instituted along with tetanus prophylaxis. Aggressive surgical debridement and drainage of pus followed by repeated minor debridement / dressing over days / weeks led to transformation of the lesion into healthy granulating wound. Wound closure was done by secondary suture, split skin graft or pedicle flap with or without unilateral orchidectomy. Patients were discharged after complete / near complete healing of scrotal wound and were followed up for 6 months. All the relevant data were collected prospectively, compiled then analyzed and our findings were compared with that in the literature. 


\section{Result}

A total of 60 patients with Fournier's gangrene treated in BIRDEM Hospital between June 2016 to March 2018 were studied. Age of the patients was from 27 to 75 years (mean age $52 \pm 4.9$ years) mostly between 40 and 60 years (Table-1).

Table -1: Age distribution of the study population

\begin{tabular}{ccc}
\hline Age (years) & Number of patients (n) & $\%$ \\
\hline$<20$ & 0 & 00 \\
$21-30$ & 02 & 3.3 \\
$31-40$ & 05 & 8.3 \\
$41-50$ & 14 & 23.4 \\
$51-60$ & 26 & 43.3 \\
$61-70$ & 10 & 16.7 \\
$>70$ & 03 & 5 \\
Total & $\mathbf{6 0}$ & $\mathbf{1 0 0}$ \\
Mean \pm SD & $\mathbf{5 2} \pm \mathbf{4 . 9}$ & \\
\hline
\end{tabular}

Among the associated risk factors, most frequent was diabetes mellitus which was present in all the cases (Table-2). In only 3 cases there was history of alcoholism (5\%).

Table-2: Associated risk factors

\begin{tabular}{lcc}
\hline Risk factor & Number of patients (n) & $\%$ \\
\hline Diabetes mellitus & 60 & 100 \\
Alcoholism & 03 & 05 \\
\hline
\end{tabular}

Duration of diabetes ranged from 0(newly diagnosed) to 22 years with mean duration of 12.3 years. On admission, in all the cases (100\%) diabetes was uncontrolled. Diabetic ketoacidosis was present in $3.3 \%$ cases. Previous glycaemic control was unsatisfactory in $93.3 \%$ cases as assessed from diabetic record book (Table 3).
Table-3: DM profile of the patients

\begin{tabular}{|c|c|c|}
\hline Variables & Number of patients (n) & $\%$ \\
\hline \multicolumn{3}{|l|}{ Duration (Years) } \\
\hline$<05$ & 09 & 15.0 \\
\hline $6-10$ & 15 & 25.0 \\
\hline $11-15$ & 25 & 40.0 \\
\hline $16-20$ & 09 & 15.0 \\
\hline$>20$ & 03 & 5.0 \\
\hline \multicolumn{3}{|l|}{ DM on Admission (RBS } \\
\hline $\mathrm{mmol} / \mathrm{L})$ & 00 & 0.0 \\
\hline$<7.8$ & 11 & 18.3 \\
\hline $7.8-<14.0$ & 31 & 51.7 \\
\hline $14.0-20.0$ & 18 & 30.0 \\
\hline$>20.0$ & 00 & 0.0 \\
\hline Controlled & 16 & 100.0 \\
\hline \multicolumn{3}{|l|}{ Uncontrolled } \\
\hline \multicolumn{3}{|l|}{ DKA on admission } \\
\hline Present & 02 & 3.3 \\
\hline Absent & 58 & 96.7 \\
\hline \multicolumn{3}{|c|}{ Previous glycaemic control } \\
\hline Satisfactory & 04 & 6.7 \\
\hline Unsatisfactory & 56 & 93.7 \\
\hline
\end{tabular}

Out of 60 cases, underlying aetiological process could be traced in $68.3 \%$ cases (Table-4). The commonest cause was local skin sepsis (36.7\%) cases, followed by anorectal sepsis in $28.3 \%$ cases. No cause could be found in $31.7 \%$ cases (Idiopathic).

Table-4: Underlying aetiological factors

\begin{tabular}{lcc}
\hline \multicolumn{1}{c}{ Aetiology } & Number of patients (n) & \% \\
\hline Idiopathic & 19 & 31.7 \\
Perianal abscess & 17 & 28.3 \\
Local skin sepsis & 22 & 36.7 \\
Periurethral abscess & $\mathbf{0 2}$ & 3.3 \\
\hline
\end{tabular}

Pus / wound discharge was taken from all 60 cases for culture and sensitivity test. $93.3 \%$ patients yielded growth of many types of organisms in varying combination (Table-5). Monomicrobial infection was detected in $31.7 \%$ cases and polymicrobial infection in $61.6 \%$ cases. Anaerobic culture was not performed. 
Table-5: Bacteriological profile

\begin{tabular}{|c|c|c|c|c|c|}
\hline \multicolumn{2}{|c|}{ Variables } & \multicolumn{2}{|c|}{ Number of patients (n) } & \multicolumn{2}{|r|}{$\%$} \\
\hline \multicolumn{2}{|c|}{ Culture negative } & \multicolumn{2}{|r|}{04} & \multicolumn{2}{|r|}{6.7} \\
\hline \multirow{2}{*}{$\begin{array}{l}\text { Culture } \\
\text { positive }\end{array}$} & Monomicrobial & \multirow{2}{*}{56} & 19 & \multirow{2}{*}{93.3} & 31.7 \\
\hline & Polymicrobial & & 37 & & $\overline{61.6}$ \\
\hline \multicolumn{6}{|c|}{ Organism isolated } \\
\hline \multicolumn{2}{|c|}{ Staphylococcus } & \multicolumn{2}{|r|}{18} & \multirow{2}{*}{\multicolumn{2}{|c|}{$\begin{array}{c}30 \\
43.3\end{array}$}} \\
\hline \multicolumn{2}{|c|}{ Streptococcus } & \multicolumn{2}{|r|}{26} & & \\
\hline \multirow{2}{*}{\multicolumn{2}{|c|}{ E. coli }} & \multicolumn{2}{|r|}{21} & \multicolumn{2}{|r|}{35} \\
\hline & & \multirow{2}{*}{\multicolumn{2}{|c|}{$\begin{array}{l}08 \\
10\end{array}$}} & \multicolumn{2}{|r|}{13.3} \\
\hline \multicolumn{2}{|c|}{$\begin{array}{l}\text { Klebsiella } \\
\text { Enterococcus }\end{array}$} & & & & 16.7 \\
\hline \multicolumn{2}{|c|}{ Pseudomonus } & \multicolumn{2}{|r|}{12} & \multicolumn{2}{|r|}{20} \\
\hline
\end{tabular}

The necrotic process had variable extension to surrounding tissues (Table-6). The scrotum was involved in all the cases. In addition, perineal extension was noted in $38.3 \%$ cases, penile in $8.3 \%$ cases, thigh and buttock in $16.7 \%$ cases and lower abdominal in $10 \%$ cases. 2 patients had very extensive tissue loss reaching up to thoracic wall and axilla.

Table-6: Area involved in necrotic process

\begin{tabular}{lcc}
\hline Area involved & Number of patients (n) & $\%$ \\
\hline Scrotum & 60 & 100 \\
Perineum & 23 & 38.3 \\
Penis & 05 & 8.3 \\
Thigh \&buttock & 10 & 16.7 \\
Lower abdominal wall & 06 & 10.0 \\
\hline
\end{tabular}

Urgent resuscitation with fluid and/ or blood transfusion was undertaken in $25(41.7 \%)$ cases. They had extensive areas of gangrene with associated toxaemia and multi-organ dysfunction. Combination injectable antibiotic (Ceftriaxone + Metronidazole) was started initially in all the cases and later on reviewed on the basis of clinical response and culture sensitivity report. Diabetes was brought under control and maintained by insulin therapy. Nutrition was maintained by enteral feeding.

Prompt debridement of necrotic skin, fascia and other non-viable tissue was done in all $100 \%$ cases (Table 7) and it had to be repeated in the majority of patients $(68.3 \%)$. On average, each patient had been debrided 1.95 times.
Table-7: Number of debridement

\begin{tabular}{ccc}
\hline Number of debridement & Number of patients (n) & \% \\
\hline 01 & 19 & 31.7 \\
02 & 29 & 48.3 \\
03 & 08 & 13.3 \\
04 & 04 & 6.7 \\
Total & $\mathbf{6 0}$ & $\mathbf{1 0 0}$ \\
\hline
\end{tabular}

In our series, five patients died during the course of treatment despite prompt initial resuscitation and urgent debridement (Table 8). 2 of them had concomitant ischemic heart disease and developed MI. In other cases, severe sepsis preceded multi organ dysfunction and failure.

Table-8: Mortality

\begin{tabular}{|c|c|c|c|c|c|}
\hline \multicolumn{2}{|c|}{ Outcome } & \multicolumn{3}{|c|}{ Number of patients (n) } & \multirow{2}{*}{$\begin{array}{c}\% \\
91.7\end{array}$} \\
\hline & vival & & 55 & & \\
\hline \multirow{2}{*}{ Death } & MODS & \multirow{2}{*}{05} & 03 & \multirow{2}{*}{8.3} & 5.0 \\
\hline & MI & & 02 & & 3.3 \\
\hline
\end{tabular}

Debridement was followed by regular dressing until healthy granulation tissue appeared ( $3-8$ weeks). Depending on the size of the wound, different techniques were applied for the closure of the wound. In the majority of the patients (50.9\%) wound closure was achieved by secondary suture with or without unilateral orchidectomy (Table 9). Unilateral orchidectomy had to be done in $7.3 \%$ cases in order to effective wound closure due to extensive scrotal tissue loss. Both testes had to be implanted in the thigh in 3 cases. Partial thickness skin graft was used to cover the defect in $34.5 \%$ cases, wither as sole technique or as an adjunct to cover the residual defect after secondary suturing. In $14.6 \%$ cases, locally based pedicle flap was done. 
Table -9: Methods of wound closure

\begin{tabular}{lcc}
\hline Technique applied & Number of patients (n) & \% \\
\hline Direct secondary closure & 24 & 43.6 \\
Secondary closure with unilateral & 04 & 7.3 \\
orchidectomy & 12 & 21.8 \\
Secondary closure with skin graft & 07 & 12.7 \\
Partial thickness skin graft & 08 & 14.6 \\
Local pedicle flap & $\mathbf{5 5}$ & $\mathbf{1 0 0}$ \\
\multicolumn{1}{c}{ Totla } &
\end{tabular}

All the patients were followed up at OPD 2 weeks after discharge and then monthly for 6 months. $23.6 \%$ patients complained of tightness of perineum on squatting and $9.1 \%$ patients complained of painful erection (Table 10) which gradually diminished over 2 to 3 months to a tolerable limit. Four patients in the unilateral orchidectomy group were psychologically upset, due to altered body image. They were treated by psychotherapy and gradually adapted to the altered body image. $7.3 \%$ patients complained of erection failure and were treated successfully with counseling and drugs. Outcome was cosmetically poor (16.4\%) particularly in those who had partial thickness skin graft as sole method. Rest of the patients of the series were found well and returned to normal social life.

Table-10: Chief complaints on follow-up

\begin{tabular}{lcc}
\hline Complaints & Number of patients (n) & \% \\
\hline Tightness of perineum & 13 & 23.6 \\
Painful erection & 05 & 9.1 \\
Psychological upset & 4 & 7.3 \\
Erectile dysfunction & 04 & 7.3 \\
Poor cosmesis & 09 & 16.4 \\
\hline
\end{tabular}

\section{Discussion}

Contrary to Fournier's specification of male youth in his description of Fournier's gangrene, there is an increasing age of patients suffering from the disease as suggested by Laor et al and Bahlmann et al. ${ }^{3}$ This is also the case in our study where the maximum numbers of patients were in the age group of 41 to 60 years $(66.7 \%)$. Far from being idiopathic, the origin of sepsis can generally be located at one of the three sites: local skin, the urinary tract and anorectal region. ${ }^{12}$ In our series, we could ascertain underlying aetiology in majority $(68.3 \%)$ of the cases.

Certain systemic conditions are frequently encountered in association with Fournier's gangrene to suggest a causal rather than a casual relationship. Diabetes has been frequently mentioned as an associated factor in the aetiology of Fournier's gangrene. ${ }^{13}$ In our series, all the patients were diabetic, which reflects the truth of the above statement. Although ketoacidosis was found in 3.3\% cases, diabetes was uncontrolled in $100 \%$ cases.

Microbiological profile of the study population suggests that in approximately $93.3 \%$ cases culture was positive for the wound swab/pus/discharge. In $61.6 \%$ patients, it was associated with poly-bacterial infection and in $31.7 \%$ cases, monobacterial infection. Streptococcus pyogens $(43.3 \%)$ was found to be the most common organism isolated in our study followed by Escherichia coli $(35 \%)$. In their experience of 38 patients, Hejase et al ${ }^{14}$ found $t$ hat $90 \%$ of the patients grew polymicrobial organisms, including gram positive and gram negative rods and gram positive cocci. The main strains grown were Staphylococcus aureus, Streptococcus, Pseudomonas, E. coli and Klebsiella. In $5 \%$ of their cases no growth was reported. Korkut et al ${ }^{15}$ had a $64 \%$ positive culture rate of the 36 patients in their case series who had cultures sent during the initial debridement, and the leading micro-organism was E. coli. In their review of 70 patients with Fournier's gangrene, Ersay et al ${ }^{16}$ found that the most frequent bacteria cultured from the wounds were E. coli (40.0\%), Bacteroids (38.6\%), Streptococcus (37.1\%), Enterococcus (27.1\%), Staphylococcus (25.7\%), Pseudomonas (24.3\%), Klebsiella $(20.0 \%)$ and proteus $(18.6 \%)$. However such infection may be the secondary complication of a primary ischaemic process. ${ }^{17}$

Treatment of Fournier's gangrene needs to be prompt and individualized. The clinical differentiation of necrotizing fasciitis from cellulitis may be difficult because the initial signs including pain, oedema and 
erythema are not distinctive. However the presence of marked toxicity out of proportion to local finding should alert the clinician. ${ }^{18}$ In severe cases with extensive area of involvement, preliminary resuscitation with intravenous fluid and/or blood transfusions should be undertaken. ${ }^{17}$ In our series, $41.7 \%$ patients required such resuscitation.

Other than scrotum (100\%), the involvement rates of perineum, thigh \& buttock, lower abdomen and penis were $31.7 \%, 15.8 \%, 10.0 \%$ and $7.5 \%$ respectively. Extensive surgical debridement to rid the patient promptly off the necrotic tissue and to prevent rapid spread of gangrene, along with treatment of predisposing conditions, is essential to achieve a cure. ${ }^{19}$ In our series, all the patients underwent major debridement to varying extent. Mean debridement time was 1.95. Diabetes was present in all cases and was controlled by short acting insulin.

Closure of the post debridement granulating wound is commonly effected by secondary suturing, as was in our series. However in cases with extensive tissue loss, closure can be effected by sacrificing one of the testicle. Otherwise, wound can be covered by partial thickness graft or local pedicle flap. Scrotal reconstruction using bilateral medial thigh fasciocutaneous flap gives the best result from aesthetic point of view.

The mortality rate averages $20 \%$ but ranges from 3 to $45 \%$. High mortality rates are found in diabetics, alcoholics and those with colorectal sources of infection who often have less typical presentation, greater delay in diagnosis and more widespread infection. ${ }^{18}$ Causes of death have included severe sepsis, caogulopathy, acute renal failure, diabetic ketoacidosis and multiple organ failure. ${ }^{17}$ In our series, mortality rate was $8.3 \%$. This may be attributed to the fact that skin was the commonest source of sepsis in our series and early diagnosis and optimal treatment was ensured through experience gained in dealing a good number of this patients on a regular basis.

\section{Conclusion}

Fournier's gangrene is both a pathological diagnosis (necrotizing fasciitis) and a clinical condition (deep perineal sepsis). The diagnosis of a Fournier's gangrene is clinical. Diabetes mellitus is frequently associated with the disease. The basic treatment of Fournier's gangrene involves prompt and aggressive extirpation of all no viable tissues, limitation and abolition of any infective process present, strict control of diabetes when present and closure of post debridement wound by either secondary closure or by skin grafting or local flap. Despite all measures, mortality of the disease may be high. Physicians should acquaint themselves with this dreadful condition to offer early and effective treatment.

\section{Conflict of interest: none.}

\section{References}

1. Patankar SP, Lalwani SK. Fournier's gangrene. J Indian Paediatr 2004; 41: 511.

2. Jones RB, Hirshmann JV, Brown GS. Necrotizing subcutaneous infection of the male genitalia. J Urol 1979; 122: 279-82

3. Bahlmann JCM. Fournier's gangrene: Necrotizing fasciitis of the male genitalia. Br J Urol. 1995; 154: 89-92.

4. Smith GL, Bunker CB, Daneen MD. Fournier's gangrene. Br J Urol 1998; 81: 347-55.

5. McLatchie GR, Leaper DJ, eds. Oxford Handbook of Clinical Surgery. 2nd ed. Oxford UK: Oxford University Press, 2003: 53, 890.

6. Muqim R. Necrotising fasciitis: management and outcome. J Coll Physicians Surg Pak 2003; 13(12): 711- 4.

7. Sudarsky LA, Laschinger JC, Coppa GF, et al. Improved results from a standardized approach in treating patients with necrotizing fasciitis. Ann Surg 1987; 206: 661-5.

8. Wong $\mathrm{CH}$, Wang YS. The diagnosis of necrotizing fasciitis. Curr Opin Infect Dis 2005; 18: 101-6.

9. Tang WM, Ho PL, Fung KK, et al. Necrotising fasciitis of a limb. J Bone Joint Surg. Br 2001; 83: 709-14

10. Sentochnik DE. Deep soft-tissue infections in diabetic patients. Infect Dis Clin North Am 1995; 9: 53-64. 
11. Anaya DA, McMahon $\mathrm{K}$, Nathens $\mathrm{AB}$, et al. Predictors of mortality and limb loss in necrotizing soft tissue infections. Arch Surg 2005; 140: 151-7.

12. S Benizri E, Fabiani P, Migliori G et al. Gangrene of the perineum. Urology 1996; 47: 935-9.

13. Nisbett AA, Thompson IM. Impact of diabetes mellitus on the presentation and outcome of Fournier's gangrene. J. Urol. 2002; 60: 775-9.

14. Hejase MJ, Simonin JE, Bihrle, et al. Genital Fournier's gangrene: Experience with 38 patients. Urology 1996; 47: 734-39.

15. Korkut M, Dayangaa M, AKG NE, Yeniay L, Erdo AN. Outcome analysis in patients with Fournier's gangrene. Disease of the colon and rectum. 2003; 46: 649-52
16. Ersay A, Yalmaz G, Akgun Y, Celik Y. Factors affecting mortality of Fournier's gangrene: Review of 70 patients. ANZ Journal of Surgery 2007; 77: 43-48

17. Eke N. Fournier's gangrene: A review of 1726 cases. Br. J. Surg. 2000; 87: 718-28

18. Campbel's Urology, 8th edition, 2002, p 591

19. Barkell DC and Villalba MR. A reappraisal of surgical management in necrotizing perianal infections. Ann Surg. 52: 395-97

20. Clayton MD, Fowler JE, Sharifi R, Pearl RK. Causes, presentation and survival of fifty seven patients with necrotizing fasciitis of the male genitalia. Surg. Gynecol Obstet 1990; 170: 49-55. 